\title{
Síndrome de burnout en estudiantes de nivel superior. Análisis comparativo entre estudiantes agrupados por programa educativo dentro del Instituto Tecnológico Superior de Ciudad Constitución
}

Burnout Syndrome in University Students. Comparative Analysis Among Students Grouped by Educational Program Within the Instituto Tecnológico Superior de Ciudad Constitución

Síndrome de Burnout em estudantes de nível superior. Análise comparativa entre estudantes agrupados por programa educacional no Instituto Superior Tecnológico da Cidade Constitución

José Ignacio Aguilar Carrasco Instituto Tecnológico Superior de Ciudad Constitución, México jaguilar@itscc.edu.mx https://orcid.org/0000-0002-5498-5752

Roberto Arvizu Acosta Instituto Tecnológico Superior de Ciudad Constitución, México rarvizu@itscc.edu.mx https://orcid.org/0000-0003-2010-6147

Ángel Evaristo Flores Ramírez Instituto Tecnológico Superior de Ciudad Constitución, México aflores@itscc.edu.mx https://orcid.org/0000-0002-6374-6585 


\section{Resumen}

En esta investigación se indagó sobre la presencia del síndrome de burnout y el nivel de este entre estudiantes inscritos en diferentes programas educativos en una institución de educación superior en México. Para ello, se aplicó un cuestionario basado en el SchoolBurnout Inventory (SBI) a 871 universitarios del Instituto Tecnológico Superior de Ciudad Constitución, quienes fueron agrupados por carrera. Posteriormente se analizaron las respuestas y se calcularon puntuaciones medias obtenidas por programa educativo. Los resultados fueron los siguientes: Arquitectura $=30.7394$; ingeniería Industrial $=30.3482$; ingeniería en Industrias Alimentarias = 29.992; licenciatura en Administración = 27.7575; ingeniería en Electromecánica $=27.5561$; ingeniería en Sistemas Computacionales $=27.3284:$ Gastronomía $=27.1443$, e ingeniería en Gestión Empresarial $=25.8775$. A partir de lo anterior se logró identificar que las puntuaciones más altas se obtuvieron en programas relacionados con el estudio de las ciencias de la ingeniería, aunque no corresponden en su totalidad a estas, ya que en la licenciatura en Administración la puntuación media obtenida se encuentra por encima de algunas ingenierías. Además, se analizó el nivel de este padecimiento entre los estudiantes matriculados exclusivamente en el programa de Arquitectura para identificar si la puntuación media aumenta conforme al grado de estudio, es decir, si el crecimiento se da uniformemente de primer hasta séptimo semestre. Los resultados indican que existe un crecimiento no uniforme, por lo tanto, no se puede reconocer que el síndrome de burnout estudiantil aumenta conforme el semestre de estudio.

Palabras clave: análisis descriptivo, educación, síndrome de burnout.

\section{Abstract}

In this research, the presence and level of burnout syndrome was identified among students enrolled in different educational programs in a higher educational institution in Mexico. For this, a questionnaire based on the School-Burnout Inventory (SBI) — adapted to Spanishwas applied to 871 university students enrolled in the Instituto Tecnológico Superior de Ciudad Constitución, who were grouped by their career. The answers were analyzed and the media obtained by educational program were calculated. The results were the following: Architecture $=30.7394$; Industrial Engineering $=30.3482$; Food Industry 
Engineering $=29.992 ;$ Bachelor of Administration $=27.7575 ;$ Electromechanical Engineering $=27.5561 ;$ Computer Systems Engineering $=27.3284 ;$ Gastronomy $=27.1443$, and Business Management Engineering $=25.8775$. It was possible to identify that the highest scores were obtained in programs related to the study of engineering sciences, although they do not fully correspond to engineering, since in the Bachelor of Administration the average score obtained is above some engineering. In addition, the level of burnout syndrome among the students enrolled in the Architecture program was analyzed to identify if the average score increases according to the semester of study, that is, if the growth increases uniformly from the first to the seventh semester. The results of this analysis indicate that there is a non-uniform growth, therefore, it cannot be recognized that student burnout syndrome increases according to the semester of study.

Keywords: descriptive analysis, education, burnout syndrome.

\section{Resumo}

Esta investigação investigou a presença da síndrome de burnout e seu nível entre estudantes matriculados em diferentes programas educacionais em uma instituição de ensino superior no México. Para isso, um questionário baseado no School-Burnout Inventory (SBI) foi aplicado a 871 estudantes universitários do Instituto Superior Tecnológico da Cidade Constitución, que foram agrupados por carreira. As respostas foram posteriormente analisadas e as pontuações médias obtidas pelo programa educacional foram calculadas. Os resultados foram os seguintes: Arquitetura $=30.7394$; Engenharia industrial $=30.3482$; Engenharia da Indústria Alimentar $=29.992$; Bacharel em Administração $=27,7575$; Engenharia eletromecânica $=27.5561$; Engenharia de sistemas de computador $=27.3284$ : Gastronomia $=27.1443$ e Engenharia de gerenciamento de negócios $=25.8775$. Pelo exposto, foi possível identificar que as pontuações mais altas foram obtidas em programas relacionados ao estudo das ciências da engenharia, embora não correspondam totalmente a essas, uma vez que, na graduação em Administração, é encontrada a pontuação média obtida acima de alguma engenharia. Além disso, o nível dessa condição foi analisado entre os alunos matriculados exclusivamente no programa Arquitetura para identificar se a pontuação média aumenta de acordo com o grau de estudo, ou seja, se o crescimento ocorre uniformemente do primeiro ao sétimo semestre. Os resultados indicam que há um 
crescimento não uniforme, portanto, não se pode reconhecer que a síndrome de burnout do aluno aumenta à medida que o semestre estudado.

Palavras-chave: análise descritiva, educação, síndrome de burnout.

Fecha Recepción: Julio 2019

Fecha Aceptación: Diciembre 2019

\section{Introducción}

El agotamiento laboral, síndrome de desgaste laboral o síndrome de burnout, denominaciones del mismo padecimiento, cobró relevancia como objeto de estudio a partir de la década de 1970, particularmente desde la disciplina de la psicología. Desde entonces ha sido un concepto que parece apuntar a una experiencia cada vez más común para las personas, $y$, por lo tanto, no ha dejado de inspirar a los investigadores a estudiarlo e intentar entender lo que es y cuáles son sus causas. Esta visibilidad del síndrome de burnout surge como resultado de una serie de cambios económicos, sociales y culturales acaecidos durante la primera mitad del siglo XX (Schaufeli et al., 2009, citados en López, 2017; Salanova y Llorens, 2008). Las primeras señales sobre su presencia se identifican durante los años 60 en Estados Unidos (Schaufeli, Leiter y Maslach, 2008), aunque, como ya se mencionó, no adquiere protagonismo hasta los años 70 (Freudenberger, 1974).

López (2017) comenta que la popularidad inicial del síndrome de burnout obedece a la descripción de las condiciones de los empleados en profesiones con alto grado de contacto con terceros. En esa misma tónica, Perlman y Hartman (1982) sugieren que esta preocupación responde a las características de las profesiones de ayuda. Maslach, Schaufeli y Leiter (2001), por su parte, refieren que estas profesiones y ocupaciones, en las cuales el objetivo es proporcionar ayuda y servicio a personas necesitadas, pueden caracterizarse como estresores emocionales e interpersonales. De esta manera, el problema del agotamiento laboral provocó llamadas a soluciones inmediatas, a pesar de la falta de un conocimiento sólido de las causas de este y correlatos. Fue así como los talleres de agotamiento se convirtieron en un modo primario de intervención, y también fueron utilizados como fuentes de datos por algunos investigadores, por ejemplo, el trabajo presentado por Pines et al. (1981, citados en Martínez (2010), titulado “Burnout, del tedio al crecimiento personal". 
El síndrome de burnout está comúnmente ligado a profesiones donde las relaciones interpersonales se presentan de manera frecuente. Freudenberger (1974) lo define como una combinación de cansancio emocional crónico, fatiga física, pérdida de interés por la actividad laboral, baja realización personal y deshumanización en el cuidado y atención de los pacientes. A partir de los años 80 se extendió su estudio hacia otras profesiones y a otros ámbitos como el militar y el industrial, hasta considerar, en 1995, a los estudiantes universitario (Uribe e Illesca, 2017, p. 235), donde a la par de las investigaciones realizadas han ido evolucionando los instrumentos para medir el nivel de dicho síndrome (Salanova y Llorens, 2008).

De forma genérica, Maslach y Leiter (1997, citados en López, 2017) consideran que la notoriedad del síndrome resulta de las políticas empresariales como consecuencia de la globalización económica. El éxito de este sistema radica en la superioridad de la productividad y la maximización de los beneficios frente al bienestar de los recursos humanos que, a la postre, se traduce en una reducción en los niveles de confianza y compromiso respecto a las organizaciones empleadoras. Lo primero que aparece en el sujeto es el cansancio emocional, lo que da paso, posteriormente, a la despersonalización y a la baja realización personal (Maslach y Leiter, 1999, citados en Serrano et al., 2017).

Así, el síndrome de burnout, también conocido como el síndrome del desgaste o de agotamiento físico y mental, constituye un problema de gran repercusión social en nuestros días.

Sin duda el interés que en la actualidad despierta ha facilitado una ampliación de su campo de estudio, pues se empezaron investigaciones en otros ámbitos profesionales y mucho más recientemente surgen los estudios con estudiantes universitarios, de entre quienes algunos logran desarrollar las competencias necesarias en su proceso formativo que favorecen la consecución de sus objetivos académicos, mientras que otros presentan dificultades que solo se registran en indicadores de elevado abandono académico (Rosales y Rosales, 2013).

En esta última situación, los estudiantes generalmente experimentan una alta carga de fatiga mental durante su proceso formativo. Algunos logran desarrollar estrategias adecuadas para afrontar las exigencias académicas, mientras que otros no lo hacen, y llegan a ser impedidos para modificar la situación problemática, lo cual trae como consecuencia el 
uso de comportamientos de escape que no son apropiadas en esta situación (Caballero, Hederich y Palacio, 2009).

Los procesos cognitivos presentes de manera natural en el contexto estudiantil, de acuerdo con Fuenmayor y Villasmil (2008), son imprescindibles para el proceso constructivo e interpretativo de la comprensión. Por ello los estudiantes de nivel superior deben de encontrar las estrategias que fomenten el aprendizaje significativo dentro y fuera de la institución educativa. Así, el estudio de los requerimientos mentales necesarios para desempeñar las actividades académicas toma gran importancia en la investigación educativa: el estudio de las causas que afectan el rendimiento escolar es una parte importante de la psicología educativa.

Durante el desarrollo de las actividades de aprendizaje en las que se encuentra involucrado el estudiante universitario pueden existir diferentes situaciones en las que la comprensión, el análisis y procesamiento de la información superan constantemente sus capacidades mentales, lo que da origen a la sobrecarga mental. Asimismo, se puede considerar que la fatiga mental en los alumnos puede estar relacionada con el exceso de trabajo intelectual, forzando a la persona continuamente a situaciones de razonamiento, solución de problemas y conceptualización que generan una carga extra de información difícil de procesar, por lo que queda en condiciones de padecer riesgos y daños psicosociales ante estos estresores y demandas concurrentes, excesivas o inapropiadas, a lo cual puede dar respuestas no adaptativas y perniciosas (Caballero, Breso y González, 2015).

Estas y otras situaciones relacionadas con el desempeño académico en los universitarios pueden contribuir a generar sensaciones de no poder dar más de sí mismo física y mentalmente, una actitud negativa de crítica (Rosales, 2012) y una desvalorización en el trabajo académico que puede facilitar la manifestación del padecimiento aquí en cuestión, el cual puede llevar al abandono escolar (Salanova y Llorens, 2008).

En el ámbito escolar existen diversas investigaciones que han identificado que los alumnos universitarios se encuentran con presiones y sobrecargas mentales propias de las actividades académicas que desempeñan. Así, el síndrome de burnout estudiantil es definido como una condición del estudiante, caracterizada, como ya se mencionó, por la sensación de no poder dar más de sí mismo, una actitud de crítica negativa, desvalorización, pérdida del interés acerca de la capacidad para realizar sus estudios 
(Schaufeli, Martínez, Pinto, Salanova y Bakker, 2002). La combinación de estos factores emocionales puede provocar bajo rendimiento escolar, lo que da origen a la desmotivación, así como a la falta de interés, razones por las cuales los estudiantes pueden tomar la decisión de abandonar sus estudios y dejar incompleta una preparación académica que les proporcionaría herramientas necesarias para afrontar las exigencias del mundo laboral. Tomando como base que la carga mental generada por las exigencias de un programa de estudio puede ser un factor que facilita la aparición del síndrome de burnout estudiantil, puede considerarse que un estudiante que se encuentra cursando una ingeniería tiene mayor probabilidad de sufrir este síndrome en comparación con un estudiante que cursa un programa con una relativa menor exigencia. En este sentido, se han encontrado diferencias en cuanto al nivel de agotamiento entre diferentes carreras (Bittar, 2008, citado en Rosales y Rosales, 2013; Martínez y Salanova, 2003, citados en Caballero et al., 2015), lo que supone un efecto por parte de las demandas de cada programa educativo.

Por otra parte, un estudio realizado en la Universidad de Granada (Balsera et al., 2016) afirma en sus resultados que el desgaste estudiantil afecta mayormente a los estudiantes que cursan niveles superiores, lo que significa que estudiantes de grados avanzados presentan mayor nivel de síndrome de burnout en comparación con sus semejantes de grados anteriores.

Considerando lo anterior, se utilizaron conocimientos básicos de estadística descriptiva para medir, analizar y divulgar la prevalencia del síndrome de burnout estudiantil empleando el cuestionario basado en el School-Burnout Inventory [SBI] (Salmela, Kiuru, Leskinen y Nurmi, 2009), adaptado al español por Boada (2015), en estudiantes matriculados en los diferentes programas educativos pertenecientes al Instituto Tecnológico Superior de Ciudad Constitución (Baja California Sur, México). Lo anterior con la finalidad de dar respuesta a las siguientes hipótesis:

1) Los participantes inscritos en los programas educativos que involucran mayor nivel de estudio de las ciencias exactas (matemáticas, física y química) presentan mayores puntuaciones en el SBI-U-9.

2) El nivel de síndrome de burnout estudiantil aumenta gradualmente conforme los semestres en el programa educativo que presenta mayor puntuación media, basados en este mismo instrumento. 


\section{Revista Iberoamericana de las Ciencias de la Salud}

Los análisis estadísticos realizados sirvieron para identificar si los estudiantes del Instituto Tecnológico Superior de Ciudad Constitución tienen una vida académica extenuante que genera una sobrecarga mental en relación con el programa educativo, y si esta sobrecarga aumenta conforme al semestre en que los participantes se encontraban matriculados.

\section{Materiales y métodos}

Para el presente trabajo se consideró a un total de 871 estudiantes matriculados en alguno de los programas de estudios ofertado por el Instituto Tecnológico Superior de Ciudad Constitución, en el estado de Baja California Sur, México, durante el semestre de agosto del 2018 a enero del 2019. En este periodo se impartían para los diferentes programas el primer, tercer, quinto y séptimo semestres, que fueron considerados así en el estudio.

El instrumento empleado fue el cuestionario SBI-U (Salmela et al., 2009), adaptado al español por Boada (2015), que consta de nueve ítems. Las respuestas para cada uno se presentan por puntuaciones en un intervalo de uno hasta seis, donde el sujeto de estudio elegirá la opción uno si está totalmente en desacuerdo con la afirmación de cada ítem, o seis si está totalmente de acuerdo con lo expresado. De esta manera, puede elegir cualquier alternativa en relación con el último mes a su situación en la universidad. Este instrumento ha mostrado buena confiabilidad y se ha aplicado en diferentes países y continentes, así como en diferentes niveles educativos (Boada, 2015).

Para realizar la aplicación se requirió del apoyo de un conjunto de estudiantes matriculados al programa educativo de ingeniería Industrial, quienes fueron capacitados sobre la intención del cuestionario y el proceso para completarse apropiadamente. Esta capacitación corrió a cargo de los docentes involucrados en la investigación. Luego de la capacitación y organizados por equipos de trabajo se distribuyeron los cuestionarios en formato papel para los diferentes grupos, considerando la disponibilidad de los sujetos de estudio en las aulas de clases. Específicamente, la aplicación implicó visitar a cada grupo, y si este se encontraba disponible y se contaba con el tiempo suficiente para responder, se procedía a la aplicación del instrumento. A cada grupo de estudio se le dio una explicación de las intenciones del cuestionario y su correcto llenado antes de entregarlo a los participantes que accedieron al estudio, ya que fue de carácter voluntario y anónimo. 
Siguiendo este procedimiento se encuestó a la totalidad de los grupos que van desde primer hasta séptimo semestre; así, se completaron todos los programas educativos con una participación por carrera como se indica en la tabla 1.

Tabla 1. Cantidad de estudiantes por programa educativo que participaron en el estudio

\begin{tabular}{|l|c|}
\hline \multicolumn{1}{|c|}{ Programa educativo } & $\begin{array}{c}\text { Cantidad de estudiantes que } \\
\text { respondieron de manera adecuada el } \\
\text { cuestionario }\end{array}$ \\
\hline Ing. Electromecánica & 154 \\
\hline Lic. en Administración de Empresas & 187 \\
\hline Ing. en Gestión Empresarial & 123 \\
\hline Ing. en Industrias Alimentarias & 56 \\
\hline Ing. Industrial & 96 \\
\hline Ing. en Sistemas Computacionales & 63 \\
\hline Arquitectura & 59 \\
\hline Gastronomía Total & 133 \\
\hline & $\mathbf{8 7 1}$ \\
\hline
\end{tabular}

Fuente: Elaboración propia

Finalizada la aplicación de cuestionarios, los resultados obtenidos se organizaron en una base de datos. Cada respuesta se vació en una hoja de cálculo Excel de Microsoft para su posterior análisis estadístico descriptivo utilizando el software SPSS.

Para cada estudiante se determinó la puntuación total de acuerdo con sus respuestas a los nueve ítems del cuestionario, y con base en estas cantidades se realizó un análisis de estadística descriptiva por carrera. Esto quiere decir que para el programa de Arquitectura se tomó la puntuación total obtenida de los 59 estudiantes mencionados en la tabla 1 y se calculó su media sin considerar la agrupación por semestres. Esta metodología se aplicó en la totalidad de los programas educativos para dar respuesta a la primera hipótesis de la presente investigación.

Para Wayne (1988), “el análisis de varianza [Anova] es un procedimiento aritmético mediante el cual la variación total de un conjunto de datos se divide en dos o más componentes, cada uno de los cuales se puede atribuir a una fuente identificable" (p. 274). Considerando esto, se decidió complementar el análisis realizando una comparación entre 
las puntuaciones medias de los programas aplicando la herramienta de Anova de un factor, empleando el software SPSS y planteando la hipótesis de igualdad de medias, que de ser rechazada habría de concluirse que las medias obtenidas por programa educativo no son significativamente iguales. En este caso, se consideró la puntuación media como la variable dependiente y el programa educativo como factor; se asignaron valores numéricos del uno a ocho a cada programa para poder cuantificar las puntuaciones por semestre, tal y como se muestra en la tabla 2. 
Tabla 2. Muestra la codificación para cada programa por semestre

\begin{tabular}{|c|c|c|c|c|c|c|c|c|}
\hline & \multicolumn{4}{|c|}{ Arquitectura (semestre $1,3,5$ y 7 ) } & \multicolumn{4}{|c|}{ Administración (semestre 1, 3, 5 y 7) } \\
\hline $\begin{array}{l}\text { Valor } \\
\text { numéric } \\
\text { o para } \\
\text { cada } \\
\text { semestre }\end{array}$ & 1 & 1 & 1 & 1 & 2 & 2 & 2 & 2 \\
\hline \multirow[t]{2}{*}{$\begin{array}{l}\text { Media } \\
\text { obtenida } \\
\text { por } \\
\text { semestre }\end{array}$} & $\begin{array}{l}28.307 \\
7\end{array}$ & $\begin{array}{l}24.733 \\
3\end{array}$ & 37.75 & $\begin{array}{l}32.166 \\
7\end{array}$ & 27.1731 & $\begin{array}{l}28.836 \\
1\end{array}$ & 28.55 & $\begin{array}{l}26.470 \\
6\end{array}$ \\
\hline & \multicolumn{4}{|c|}{ Ing. Industrial (semestre 1, 3, 5 y 7) } & \multicolumn{4}{|c|}{$\begin{array}{c}\text { Ing. en Industrias Alimentarias } \\
\text { (semestre } 1,3,5 \text { y 7) }\end{array}$} \\
\hline $\begin{array}{l}\text { Valor } \\
\text { numéric } \\
\text { o para } \\
\text { cada } \\
\text { semestre }\end{array}$ & 3 & 3 & 3 & 3 & 4 & 4 & 4 & 4 \\
\hline \multirow[t]{2}{*}{$\begin{array}{l}\text { Media } \\
\text { obtenida } \\
\text { por } \\
\text { semestre }\end{array}$} & $\begin{array}{l}27.390 \\
2\end{array}$ & 27.28 & $\begin{array}{l}34.631 \\
6\end{array}$ & $\begin{array}{l}32.090 \\
9\end{array}$ & 26.4375 & $\begin{array}{l}34.187 \\
5\end{array}$ & 33.2 & $\begin{array}{l}26.142 \\
9\end{array}$ \\
\hline & \multicolumn{4}{|c|}{$\begin{array}{c}\text { Ing. en Sistemas computacionales } \\
\text { (semestre } 1,3,5 \text { y } 7)\end{array}$} & \multicolumn{4}{|c|}{$\begin{array}{c}\text { Ing. en Gestión Empresarial } \\
\text { (semestre } 1,3,5 \text { y 7) }\end{array}$} \\
\hline $\begin{array}{l}\text { Valor } \\
\text { numéric } \\
\text { o para } \\
\text { cada } \\
\text { semestre }\end{array}$ & 5 & 5 & 5 & 5 & 6 & 6 & 6 & 6 \\
\hline $\begin{array}{l}\text { Media } \\
\text { obtenida }\end{array}$ & 28.68 & 26 & $\begin{array}{l}27.166 \\
7\end{array}$ & $\begin{array}{l}27.466 \\
7\end{array}$ & 25 & $\begin{array}{l}24.782 \\
6\end{array}$ & $\begin{array}{l}26.727 \\
3\end{array}$ & 27 \\
\hline
\end{tabular}




\begin{tabular}{|c|c|c|c|c|c|c|c|c|}
\hline \multirow[t]{2}{*}{$\begin{array}{l}\text { por } \\
\text { semestre }\end{array}$} & & & & & & & & \\
\hline & \multicolumn{4}{|c|}{$\begin{array}{l}\text { Ing. en Electromecánica (semestre } \\
\qquad 1,3,5 \text { y } 7)\end{array}$} & \multicolumn{4}{|c|}{ Gastronomía (semestre $1,3,5$ y 7) } \\
\hline \begin{tabular}{|l} 
Valor \\
numéric \\
o para \\
cada \\
semestre
\end{tabular} & 7 & 7 & 7 & 7 & 8 & 8 & 8 & 8 \\
\hline $\begin{array}{l}\text { Media } \\
\text { obtenida } \\
\text { por } \\
\text { semestre }\end{array}$ & 27.16 & $\begin{array}{l}26.343 \\
8\end{array}$ & $\begin{array}{l}28.363 \\
6\end{array}$ & $\begin{array}{l}28.357 \\
1\end{array}$ & 26.34 & $\begin{array}{l}26.230 \\
8\end{array}$ & $\begin{array}{l}28.406 \\
3\end{array}$ & 27.6 \\
\hline
\end{tabular}

Fuente: Elaboración propia

Para conocer si el síndrome de burnout aumenta de manera gradual se realizó una comparación entre las puntuaciones medias obtenidas en los estudiantes participantes agrupados por semestres (primero, tercero, quinto y séptimo) del programa educativo que resultó tener la mayor puntuación general y, utilizando el software SPSS, se obtuvo el coeficiente de correlación de Pearson para conocer la relación entre el semestre cursado y su puntuación obtenida. Con esto se respondió a la segunda hipótesis de la presente investigación.

\section{Resultados}

Para comprobar la primera hipótesis, las puntuaciones de los participantes se ordenaron por programa educativo. En cada caso se sumó la puntuación de las respuestas por estudiante y se obtuvo una media. La carrera de Arquitectura, con 30.7394, obtuvo la puntuación media más alta y la menor se obtuvo en ingeniería en Gestión Empresarial, con 25.8775 (ver la tabla 3 y la figura 1). Los descriptivos generales obtenidos mediante el programa SPSS se muestran en la tabla 4. 
Tabla 3. Puntuaciones medias obtenidas por programa educativo en el periodo de estudio, luego de tener la puntuación total por estudiante matriculado en los programas incluidos

\begin{tabular}{|c|c|c|c|c|c|c|c|c|}
\hline $\begin{array}{l}\text { Programa } \\
\text { educativo }\end{array}$ & 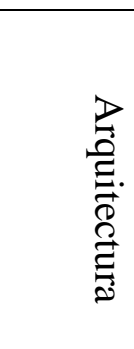 & 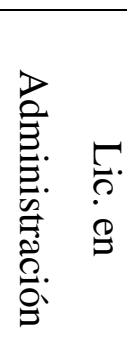 & 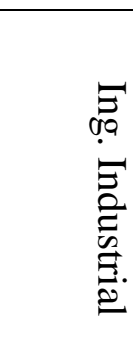 & 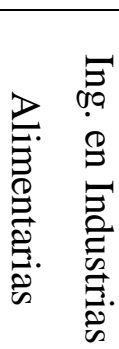 & 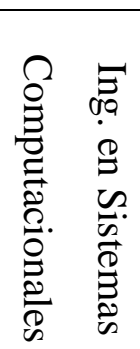 &  & 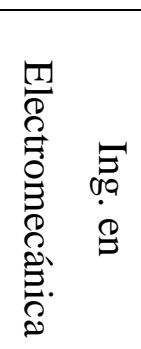 & 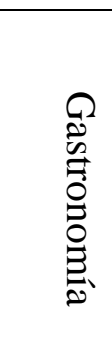 \\
\hline Puntuació & 30.739 & 27.757 & 30.348 & 29.99 & 27.328 & 25.877 & 27.556 & 27.14 \\
\hline $\begin{array}{l}\text { n media } \\
\text { obtenida }\end{array}$ & 4 & 5 & 2 & 2 & 4 & 5 & 1 & 43 \\
\hline
\end{tabular}

Fuente: Elaboración propia

Figura 1. Diferencia en las puntuaciones obtenidas por programa educativo

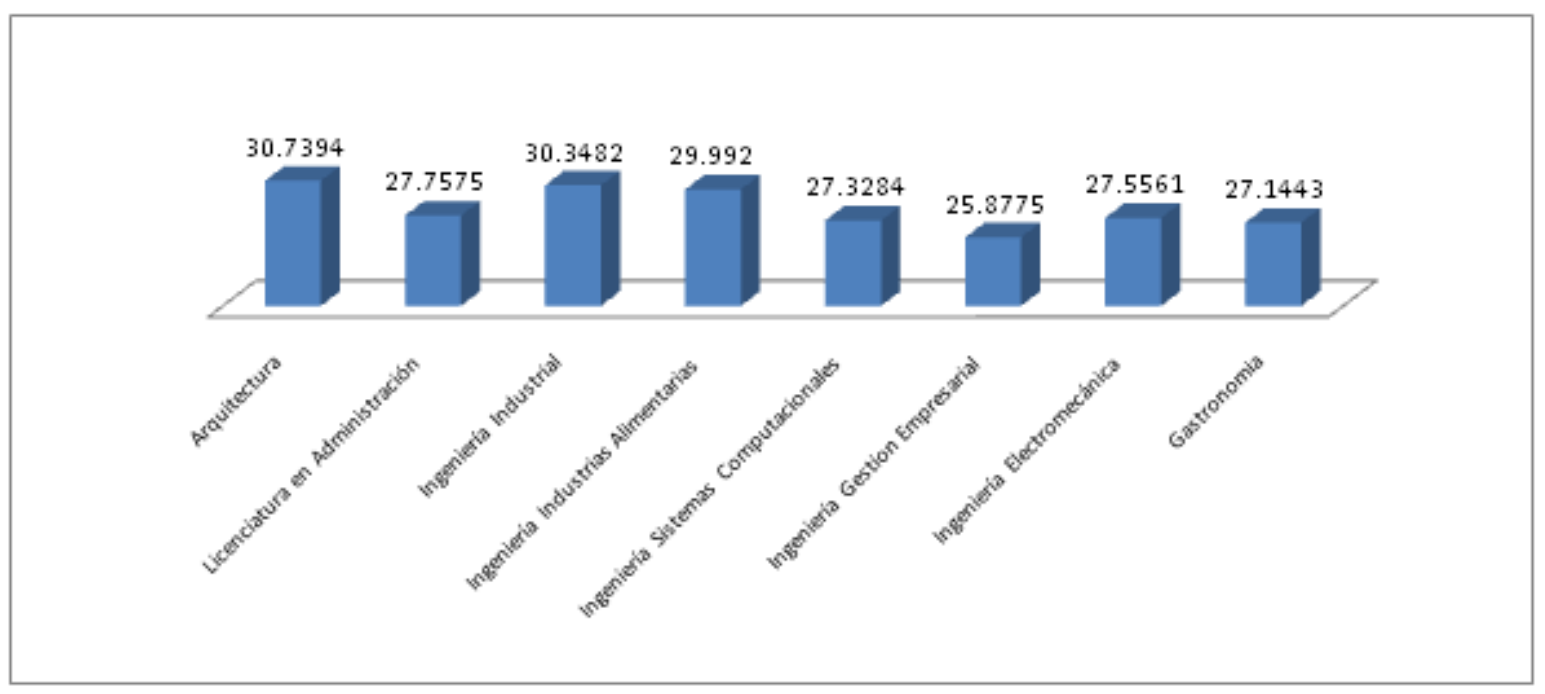

Fuente: Elaboración propia 
Revista Iberoamericana de las Ciencias de la Salud

Tabla 4. Estadísticos descriptivos obtenidos mediante el programa SPSS

\begin{tabular}{|c|c|c|c|c|c|c|c|c|}
\hline \multirow[t]{2}{*}{$\begin{array}{l}\text { Programa } \\
\text { educativo }\end{array}$} & \multirow{2}{*}{$\begin{array}{c}\mathbf{N} \\
\\
\text { Límit } \\
\mathrm{e} \\
\text { inferi } \\
\text { or }\end{array}$} & \multirow{2}{*}{\begin{tabular}{|c|} 
Media \\
Límite \\
superio \\
r
\end{tabular}} & \multirow{2}{*}{$\begin{array}{c}\text { Desviac } \\
\text { ión } \\
\text { típica } \\
\text { Límite } \\
\text { inferior }\end{array}$} & \multirow{2}{*}{$\begin{array}{c}\text { Error } \\
\text { típico } \\
\text { Límite } \\
\text { superio } \\
\text { r }\end{array}$} & \multicolumn{2}{|c|}{$\begin{array}{c}\text { Intervalo de } \\
\text { confianza para la } \\
\text { media al } 95 \%\end{array}$} & \multirow{2}{*}{$\begin{array}{c}\text { Míni } \\
\text { mo } \\
\text { Límit } \\
\text { e } \\
\text { inferi } \\
\text { or }\end{array}$} & \multirow{2}{*}{$\begin{array}{c}\text { Máxi } \\
\text { mo } \\
\text { Límite } \\
\text { superi } \\
\text { or }\end{array}$} \\
\hline & & & & & $\begin{array}{l}\text { Límite } \\
\text { inferior }\end{array}$ & $\begin{array}{l}\text { Límite } \\
\text { superio } \\
\mathrm{r}\end{array}$ & & \\
\hline Arc & 4 & $\begin{array}{r}30.7394 \\
25\end{array}$ & $\begin{array}{r}5.57291 \\
37\end{array}$ & $\begin{array}{r}2.78645 \\
68\end{array}$ & $\begin{array}{r}21.8716 \\
76\end{array}$ & $\begin{array}{r}39.6071 \\
74\end{array}$ & $\begin{array}{r}24.73 \\
33\end{array}$ & $\begin{array}{r}37.75 \\
00\end{array}$ \\
\hline $\begin{array}{l}\text { Lic. en } \\
\text { Administraci } \\
\text { ón }\end{array}$ & 4 & $\begin{array}{r}27.7574 \\
50\end{array}$ & $\begin{array}{r}1.12384 \\
30\end{array}$ & $\begin{array}{r}.561921 \\
5\end{array}$ & $\begin{array}{r}25.9691 \\
65\end{array}$ & $\begin{array}{r}29.5457 \\
35\end{array}$ & $\begin{array}{r}26.47 \\
06\end{array}$ & $\begin{array}{r}28.83 \\
61\end{array}$ \\
\hline $\begin{array}{l}\text { Ing. } \\
\text { Industrial }\end{array}$ & 4 & $\begin{array}{r}30.3481 \\
75\end{array}$ & $\begin{array}{r}3.63080 \\
04\end{array}$ & $\begin{array}{r}1.81540 \\
02\end{array}$ & $\begin{array}{r}24.5707 \\
61\end{array}$ & $\begin{array}{r}36.1255 \\
89\end{array}$ & $\begin{array}{r}27.28 \\
00\end{array}$ & $\begin{array}{r}34.63 \\
16\end{array}$ \\
\hline $\begin{array}{l}\text { Ing. en } \\
\text { Industrias } \\
\text { Alimentarias }\end{array}$ & 4 & $\begin{array}{r}29.9919 \\
75\end{array}$ & $\begin{array}{r}4.29509 \\
51\end{array}$ & $\begin{array}{r}2.14754 \\
75\end{array}$ & $\begin{array}{r}23.1575 \\
20\end{array}$ & $\begin{array}{r}36.8264 \\
30\end{array}$ & $\begin{array}{r}26.14 \\
29\end{array}$ & $\begin{array}{r}34.18 \\
75\end{array}$ \\
\hline $\begin{array}{l}\text { Ing. en } \\
\text { Sistemas } \\
\text { Computacio } \\
\text { nales }\end{array}$ & 4 & $\begin{array}{r}27.3283 \\
50\end{array}$ & $\begin{array}{r}1.10102 \\
12\end{array}$ & $\begin{array}{r}.550510 \\
6\end{array}$ & $\begin{array}{r}25.5763 \\
80\end{array}$ & $\begin{array}{r}29.0803 \\
20\end{array}$ & $\begin{array}{r}26.00 \\
00\end{array}$ & $\begin{array}{r}28.68 \\
00\end{array}$ \\
\hline $\begin{array}{l}\text { Ing. en } \\
\text { Gestión } \\
\text { Empresarial }\end{array}$ & 4 & $\begin{array}{r}25.8774 \\
75\end{array}$ & $\begin{array}{r}1.14760 \\
31\end{array}$ & $\begin{array}{r}.573801 \\
5\end{array}$ & $\begin{array}{r}24.0513 \\
82\end{array}$ & $\begin{array}{r}27.7035 \\
68\end{array}$ & $\begin{array}{r}24.78 \\
26\end{array}$ & $\begin{array}{r}27.00 \\
00\end{array}$ \\
\hline $\begin{array}{l}\text { Ing. en } \\
\text { Electromecá } \\
\text { nica }\end{array}$ & 4 & $\begin{array}{r}27.5561 \\
25\end{array}$ & $\begin{array}{r}.986614 \\
4\end{array}$ & $\begin{array}{r}.493307 \\
2\end{array}$ & $\begin{array}{r}25.9862 \\
01\end{array}$ & $\begin{array}{r}29.1260 \\
49\end{array}$ & $\begin{array}{r}26.34 \\
38\end{array}$ & $\begin{array}{r}28.36 \\
36\end{array}$ \\
\hline Gastronomía & 4 & $\begin{array}{r}27.1442 \\
75\end{array}$ & $\begin{array}{r}1.04589 \\
47\end{array}$ & $\begin{array}{r}.522947 \\
4\end{array}$ & $\begin{array}{r}25.4800 \\
23\end{array}$ & $\begin{array}{r}28.8085 \\
27\end{array}$ & $\begin{array}{r}26.23 \\
08\end{array}$ & $\begin{array}{r}28.40 \\
63\end{array}$ \\
\hline Total & 32 & 28.3429 & 3.07750 & .544031 & 27.2333 & 29.4524 & 24.73 & 37.75 \\
\hline
\end{tabular}




\begin{tabular}{|l|l|l|l|l|l|l|l|l|}
\hline & & 06 & 68 & 5 & 47 & 66 & 33 & 00 \\
\hline
\end{tabular}

Fuente: Elaboración propia

Los resultados del Anova unifactorial mostraron un nivel de significación de 0.226, como se muestra en la tabla 5. Por lo tanto, fue innecesario realizar el análisis de comparaciones múltiples, ya que $0.226>0.05$; así se demostró que la hipótesis de igualdad de medias no se rechaza, y es posible afirmar que no existen diferencias significativas entre las puntuaciones medias obtenidas por programa educativo.

Tabla 5. Resultados del procedimiento Anova de un factor utilizando el programa SPSS

\begin{tabular}{|l|r|r|r|r|l|}
\hline & Suma de cuadrados & \multicolumn{1}{|c|}{ gl } & Media cuadrática & F & Sig. \\
\hline Intergrupos & 87.960 & 7 & 12.566 & 1.467 & 0.226 \\
\hline Intragrupos & 205.642 & 24 & 8.568 & & \\
\hline Total & 293.602 & 31 & & & \\
\hline
\end{tabular}

Fuente: Elaboración propia

En relación con la hipótesis número dos, debido a que obtuvo la mayor puntuación media (30.7394), se consideró a la carrera de Arquitectura. Para identificar si existe un aumento gradual en la puntuación media de primer a séptimo semestre, los resultados se clasificaron según el semestre en el que los participantes se encontraban matriculados al momento de la aplicación de los cuestionarios (tabla 6 y figura 2).

Tabla 6. Puntuaciones medias obtenidas por semestre en el periodo de estudio para el programa de Arquitectura

\begin{tabular}{|l|c|c|c|c|}
\hline & $\begin{array}{c}\text { Primer } \\
\text { semestre }\end{array}$ & $\begin{array}{c}\text { Tercer } \\
\text { semestre }\end{array}$ & $\begin{array}{c}\text { Quinto } \\
\text { semestre }\end{array}$ & $\begin{array}{c}\text { Séptimo } \\
\text { semestre }\end{array}$ \\
\hline $\begin{array}{l}\text { Puntuación } \\
\text { media } \\
\text { obtenida }\end{array}$ & 28.3077 & 24.7333 & 37.75 & 32.1667 \\
\hline
\end{tabular}

Fuente: Elaboración propia 
Figura 2. Puntuaciones obtenidas por semestre del programa educativo de Arquitectura

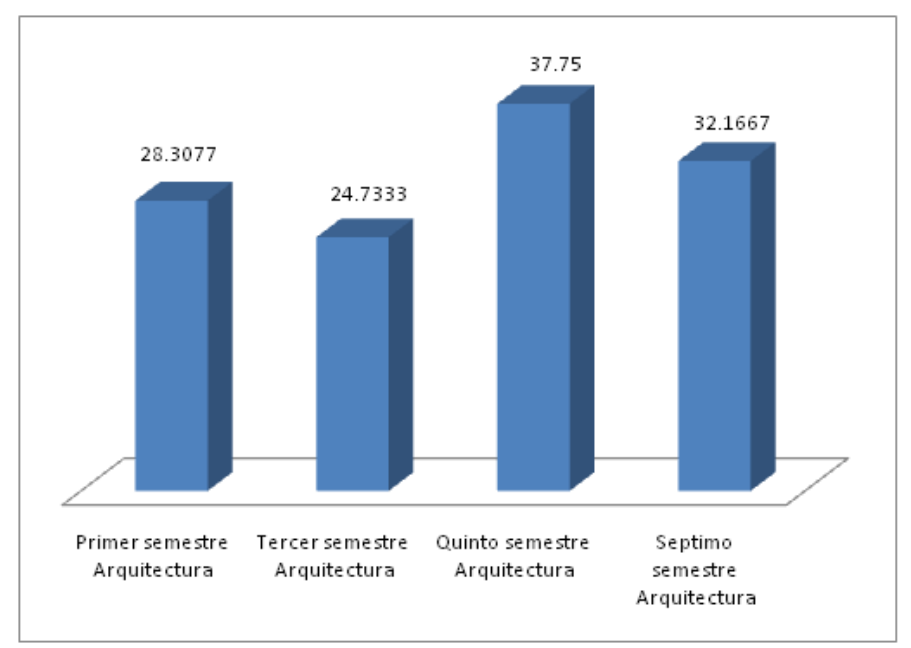

Fuente: Elaboración propia

Para complementar la comprobación de esta hipótesis se calculó el coeficiente de correlación de Pearson, empleando la herramienta de análisis de correlación bivariada de SPSS. De esta forma se obtuvo un valor de 0.570 en la relación entre las variables Semestre y Puntuación media con una significancia bilateral de 0.430 , tal y como se muestra en la tabla 7, lo que indica que no se permite rechazar la hipótesis nula (sig. > 0.05), es decir, no existe relación entre las variables, por lo tanto, no existe un crecimiento gradual (como se observa en la figura 2) entre las puntuaciones medias obtenidas de los semestres del programa de Arquitectura.

Tabla 7. Resultados del coeficiente de correlación de Pearson para las variables Semestre y Puntuación media, utilizando el programa SPSS

\begin{tabular}{|l|l|r|r|}
\hline & & Semestre & \multicolumn{2}{|c|}{$\begin{array}{c}\text { Puntuación } \\
\text { media }\end{array}$} \\
\hline Semestre & Correlación de Pearson & 1 & 0.570 \\
\hline & Sig. (bilateral) & 4 & 0.430 \\
\hline $\begin{array}{l}\text { Puntuación } \\
\text { media }\end{array}$ & N & 0.570 & 4 \\
\hline & Sig. (bilateral) & 0.430 & \\
\hline & N & 4 & 4 \\
\hline
\end{tabular}

Fuente: Elaboración propia 


\section{Discusión}

Considerando los resultados obtenidos en la comparación de puntuaciones medias de los sujetos de estudio agrupados por programa educativo (tabla 3), no existe diferencia significativa entre las puntuaciones medias por programa educativo, pero sí se manifiesta una tendencia tomando los más altos, Arquitectura e ingeniería Industrial, con puntuación similar, que están por encima de otros programas de carácter económico-administrativo, como licenciatura en Administración e ingeniería en Gestión Empresarial. Entrando en el terreno de la especulación, se podría decir que uno de los motivos de estos resultados puede ser la exigencia de las actividades académicas que involucran mayor comprensión de las asignaturas relacionadas con las matemáticas, ya que en el caso de la formación de los ingenieros se dice que estos dedican cerca de $80 \%$ del tiempo de estudio a las ciencias básicas, a las ciencias de la ingeniería y a la ingeniería aplicada (Cantoral, 2001), así como una mayor necesidad de concentrarse por más tiempo en las actividades académicas correspondientes. Se sugiere realizar futuras investigaciones sobre este punto para verificar las causas, ya que, como se menciona, dado la carga que presentan, existen excesos de trabajo en los estudiantes, por las diferentes condiciones en que se desenvuelven, que provocan el síndrome de burnout (Barraza, 2008, 2011, citado en Uribe e Illesca, 2017; Caballero et al., 2015; Rosales y Rosales, 2013).

Teniendo en cuenta que la hipótesis de igualdad de medias considerada en el Anova unifactorial fue aceptada, como se demostró en los resultados, existe similitud entre las puntuaciones medias de los programas educativos analizados. Esto se presenta con mayor notoriedad en los programas de licenciatura en Administración, Gastronomía, ingeniería Electromecánica e ingeniería en Sistemas Computacionales. Se puede deducir que los estudiantes matriculados en dichas carreras presentan niveles similares de agotamiento a pesar de que los contenidos temáticos de las asignaturas que integran cada una de ellas difieren en relación con el estudio de las ciencias de la ingeniería. Esta condición puede resultar de una combinación de circunstancias que rodean a los estudiantes, incluyendo, como mencionan Barradas, Trujillo, Sánchez y López (2017), la evaluación del maestro, la sobrecarga de trabajo, las exposiciones en el aula, la elaboración de ensayos o trabajos de investigación, la tensión con sus profesores, factores considerados por los propios estudiantes como estresores, todo lo cual puede resultar en síndrome de burnout. Por otra parte, una baja dedicación al estudio puede llevar al estudiante a sentir mayor tensión en las 
evaluaciones, lo que lleva también a la presencia del mencionado síndrome (Novoa, Burgos, Zentner y Toro, 2018). De esta manera, la similitud de desgaste entre carreras con diferente nivel de exigencia desde el punto de vista de las ciencias exactas podría explicarse.

En general, se pueden ordenar los resultados de manera decreciente para identificar cuáles son los programas con mayores niveles de agotamiento: Arquitectura = 30.7394; ingeniería Industrial $=30.3482$; ingeniería en Industrias Alimentarias $=29.992$; licenciatura en Administración $=27.7575$; ingeniería en Electromecánica $=27.5561$; ingeniería en Sistemas Computacionales $=27.3284$; Gastronomía $=27.1443$, e ingeniería en Gestión Empresarial $=25.8775$. Otros estudios coinciden con las diferencias de síndrome de burnout entre carreras (Bittar, 2008, citado en Rosales y Rosales, 2013; Martínez y Salanova, 2003, citados en Caballero et al., 2015).

Por otro lado, tomando en cuenta que las personas mayores tienen más posibilidades de sufrir este padecimiento (del Río, Rivera, Rueda, Serrano y Villalta, 2015), se consideró la hipótesis de que los estudiantes de grados avanzados presentarían niveles de agotamiento más elevados. Para comprobarlo, se seleccionó a los estudiantes cuyo programa educativo presentó mayor puntuación media, a saber, el programa de Arquitectura. Como se demostró anteriormente y se puede observar en la figura 2, no existe un crecimiento ascendente uniforme entre los estudiantes agrupados de primer a séptimo semestre. Además, es importante señalar que los alumnos de primer semestre no fueron quienes obtuvieron la menor puntuación, ya que se ubican por debajo de quinto y séptimo semestre. Esto se comprueba mediante el análisis de correlación realizado en la tabla 7, donde se demostró con el valor de la significación que las variables Semestre y Puntuación media no están relacionadas entre sí. En conclusión, no se puede afirmar que el síndrome de burnout aumenta o disminuye entre los estudiantes de Arquitectura conforme el grado cursado. Esto puede sugerir que la exigencia de la educación superior puede infligir mayor preocupación en los jóvenes de nuevo ingreso.

Al respecto, en la investigación de González y Abarca (2017) se menciona que parte del proceso de adaptación involucra realizar actividades que en la educación media superior no eran muy frecuentes, como aprender a ser autosuficientes, hacer citas en textos, desvelarse estudiando y aplicar nuevas estrategias de aprendizaje. Por lo tanto, su estado emocional puede verse afectado durante el proceso transición de educación media superior 
a nivel superior. Es probable que al desarrollar una carrera la exigencia no sea gradual, o al menos la percepción de exigencia del estudiante en su adaptación y desarrollo en el nivel superior no sea gradual, es decir, no va sintiendo mayor exigencia conforme avanza en el plan de estudios, sino que ciertos semestres, como en este caso el primero y el quinto, le representen una exigencia mayor. Coincidentemente, Uribe e Illesca (2017), en un esfuerzo por comprobar que la sobrecarga de demandas estudiantiles es un riesgo importante para desarrollar un estrés prolongado, obtuvieron como resultado un agotamiento físico, cognitivo y emocional, y validaron así la definición del síndrome de burnout académico. Según estudios, el primer y quinto semestre son los que despuntan como los más críticos al momento de afrontar el estrés, situación que se deriva de las demandas académicas y sociales (Uribe e Illesca, 2017). Como se observa en la tabla 6, la puntuación más baja se obtuvo en los estudiantes de tercer semestre, lo que sugiere que en ese grado se encuentran mejor adaptados que al inicio de su formación académica. Esto puede suponer un relajamiento gracias a una mejor adaptación a las exigencias de la educación superior. Puede ser en este punto cuando algunos estudiantes logran desarrollar estrategias adecuadas para afrontar las exigencias académicas (Rosales y Rosales, 2013). Considerando lo anterior, la segunda hipótesis planteada no puede ser aceptada, ya que los niveles de las variables (puntuación media y semestre) no presentan relación directa. Asimismo, no es visible un aumento gradual conforme los estudiantes avanzan de semestre. 


\section{Conclusiones}

Conforme al análisis de los resultados obtenidos, se presenta una diferencia, aunque no significativa estadísticamente hablando, entre programas educativos en cuanto al nivel de síndrome de burnout que presentan los estudiantes, lo que deriva de las exigencias que conlleva la vida estudiantil con factores como las evaluaciones, la preparación de tareas, proyectos y exámenes, la relación con los profesores y los horarios. Se puede observar en la tabla 3 del presente estudio que el nivel de agotamiento es mayor en la carrera de Arquitectura, que no es catalogada como una ingeniería. No obstante, como se demuestra en la retícula (Instituto Tecnológico Superior de Ciudad Constitución, s. f.), los contenidos de las asignaturas tienen una relación significativa con los contenidos de las asignaturas de ciencias exactas aplicadas a la ingeniería. En este contexto, se puede concluir que esta carrera tiene niveles similares a los obtenidos en las ingenierías del Instituto Tecnológico Superior de Ciudad Constitución, dados los contenidos y sumado a esto las presiones propias de la vida estudiantil.

Asimismo, se analizó el crecimiento de los niveles del síndrome de burnout conforme el aumento de grado de estudios, tomando en cuenta a la carrera de Arquitectura, y se determinó que el mayor nivel se presenta en el primero y quinto semestre, lo que coincide con otros estudios. Con esto se demuestra que cada semestre puede tener sus particularidades que lo hacen distinto en relación con las exigencias académicas que el estudiante afronta sin importar el nivel de avance reticular. 


\section{Referencias}

Balsera, M., Chavasco, G., López, R., Pérez, A., Sánchez, M. y Villena, C. (2016). Estudio del Burnout en estudiantes de la Universidad de Granada. Influencia moduladora de variables de inteligencia emocional y clasificación por cursos. ReiDoCrea, 5(2), 2732. Recuperado de http://digibug.ugr.es/bitstream/handle/10481/41430/5-25.pdf? sequence $=1 \&$ isAllowed $=\mathrm{y}$.

Barradas, M., Trujillo, G., Sánchez, A. V. y López, J. (2017). Burnout estudiantil en universitarios veracruzanos. RIDE Revista Iberoamericana para la Investigación y el Desarrollo Educativo, 7(14). Recuperado de http://www.redalyc.org/articulo.oa?id=498153999002.

Boada, J., Merino, E., Sánchez, J., Prizmic, A. and Vigil, A. (2015). Adaptation and psychometric properties of the SBI-U scale for Academic Burnout in university students. Anales de Psicología, 31(1), 290-297. Retrieved from https://revistas.um.es/analesps/article/view/analesps.31.1.168581/169821.

Caballero, C., Breso, É. y González, O. (2015). Burnout en estudiantes universitarios. Psicología desde el Caribe, 32(3), 424-441. Recuperado de http://www.redalyc.org/articulo.oa?id=21342681007.

Caballero, C., Hederich, C. y Palacio J. (2009). El Burnout Académico: Delimitación del Síndrome y factores asociados con su aparición. Revista Latinoamericana de Psicología, 42, 131-146. Recuperado de https://www.redalyc.org/pdf/805/80515880012.pdf.

Cantoral, R. (2001). Enseñanza de la matemática en la educación superior. Sinéctica, Revista Electrónica de Educación, (19), 3-27. Recuperado de https://www.redalyc.org/pdf/998/99817935002.pdf.

Del Río, C., Rivera, M., Rueda, C., Serrano, M. D. y Villalta, I. (2015). Burnout en relación con el equipo de celadores del Hospital Virgen de las Nieves. ReiDoCrea, 4, 144150. http://digibug.ugr.es/bitstream/handle/10481/37028/ReiDoCrea-Vol.4Art.21.pdf? sequence $=1 \&$ isAllowed $=\mathrm{y}$.

Freudenberger, H. J. (1974). Staff Burn-Out. Journal of Social Issues, 30(1), 159-165. Retrieved from https://spssi.onlinelibrary.wiley.com/doi/abs/10.1111/j.15404560.1974.tb00706.x. 
Fuenmayor, G. y Villasmil, Y. (2008). La percepción, la atención y la memoria como procesos cognitivos utilizados para la comprensión textual. Revista de Artes y Humanidades UNICA, 9(22), 187-202. Recuperado de http://www.redalyc.org/articulo.oa?id=170118859011.González, R. y Abarca, M. (2017). La transición del estudiante universitario el primer año de estudios en la Universidad de Colima. Colima, México: Universidad de Colima.

Instituto Tecnológico Superior de Ciudad Constitución (s. f.). Arquitectura. Recuperado de somoshalcones.com/index.php/carreras/arquitectura.

López, A. (2017). El Síndrome de Burnout: Antecedentes y consecuentes organizacionales en el ámbito de la sanidad pública gallega. (tesis doctoral). Universidad de Vigo, España. Recuperado de http://www.investigo.biblioteca.uvigo.es/xmlui/bitstream/handle/11093/791/sindro me_burn out_antecedentes_consecuentes_organizacionales_sanidad_gallega_analia.pdf?sequ ence $=1$.

Martínez, A. (2010). El Síndrome de Burnout. Evolución conceptual y estado actual de la cuestión. Vivat Academia, (112), 42-80. Recuperado de https://www.redalyc.org/articulo.oa?id=5257/525752962004.

Maslach, C., Schaufeli, W. B. and Leiter, M. (2001). Job Burnout. Annual Review of Psychology, 52, 397-422. Retrieved from https://www.wilmarschaufeli.nl/publications/Schaufeli/154.pdf.

Novoa, P., Burgos, R., Zentner, J. y Toro, C. (2018). Burnout en estudiantes de enfermería de una Universidad peruana. Investigación en Educación Médica, 7(26), 111-113. Recuperado de http://www.redalyc.org/articulo.oa?id=349759847014.

Perlman, B. and Hartman, E. A. (1982). Burnout - Summary and Future-Research. Human Relations, 35(4), 283-305. Recuperado de https://files.eric.ed.gov/fulltext/ED205840.pdf.

Rosales, Y. (2012). Estudio unidimensional del síndrome de burnout en estudiantes de medicina de Holguín. Revista de la Asociación Española de Neuropsiquiatría, 32(116), 787-793. Recuperado de http://www.revistaaen.es/index.php/aen/article/view/16574. 
Rosales, Y. y Rosales, F. R. (2013). Burnout estudiantil universitario. Conceptualización y estudio. Salud Mental, 36(4), 337-345. Recuperado de http://www.redalyc.org/articulo.oa?id=58228969009.

Salanova, M. y Llorens, S. (2008). Estado actual y retos futuros en el estudio del burnout. Papeles del Psicólogo, 29(1), 59-67. Recuperado de http://www.redalyc.org/articulo.oa?id=77829108.Schaufeli, W. B., Leiter, M. P. and Maslach, C. (2008). Burnout: 35 years of research and practice. Career Development International, 14(3), 204-220. Retrieved from https://www.wilmarschaufeli.nl/publications/Schaufeli/311.pdf.

Schaufeli, W. B., Martínez, I., Pinto, A. M., Salanova, M. and Bakker, A. (2002). Burnout and engagement in university students: A cross-national study. Journal of CrossCultural Psychology, 33(5), 464-481. Retrieved from https://www.isonderhouden.nl/doc/pdf/arnoldbakker/articles/articles_arnold_bakker _78.pdf.Salmela, K., Kiuru, N., Leskinen, E. and Nurmi, J. E. (2009). SchoolBurnout Inventory (SBI) - Reliability and validity. European Journal of Psychological Assessment, 25(1), 48-57. Retrieved from https://www.researchgate.net/publication/232593343_School_Burnout_Inventory_S BI_Reliability_and_Validity.

Serrano, M. A. Rangel, A., Vidal, C., Ureña, Y., Anillo, H. y Angulo, G. (2017). Burnout. Síndrome silencioso que afecta el desempeño laboral de los docentes universitarios. Revista Espacios, $38(55) . \quad$ Recuperado de https://www.revistaespacios.com/a17v38n55/a17v38n55p28.pdf.

Uribe, M. A. e Illesca, M. (2017). Burnout en estudiantes de enfermería de una universidad privada. Investigación en Educación Médica, 6(24), 234-241. Recuperado de http://www.redalyc.org/articulo.oa?id=349753310004.

Wayne, D. (1988). Estadística con aplicaciones a las ciencias sociales y a la educación. Estado de México, México: McGraw-Hill.

\begin{tabular}{|l|l|}
\hline Rol de Contribución & Autor (es) \\
\hline Conceptualización & $\begin{array}{l}\text { José Ignacio Aguilar Carrasco «principal», Roberto Arvizu } \\
\text { Acosta «apoyo», }\end{array}$ \\
\hline Metodología & José Ignacio Aguilar Carrasco \\
\hline
\end{tabular}




\begin{tabular}{|l|l|}
\hline Software & José Ignacio Aguilar Carrasco \\
\hline Validación & $\begin{array}{l}\text { José Ignacio Aguilar Carrasco «igual», Roberto Arvizu Acosta } \\
\text { «igual», Ángel Evaristo Flores Ramírez «apoyo» }\end{array}$ \\
\hline Análisis Formal & $\begin{array}{l}\text { José Ignacio Aguilar Carrasco «igual», Roberto Arvizu Acosta } \\
\text { «igual», }\end{array}$ \\
\hline Investigación & José Ignacio Aguilar Carrasco \\
\hline Recursos & Ángel Evaristo Flores Ramírez \\
\hline Curación de datos & $\begin{array}{l}\text { José Ignacio Aguilar Carrasco «igual», Roberto Arvizu Acosta } \\
\text { «igual», Ángel Evaristo Flores Ramírez «igual» }\end{array}$ \\
\hline $\begin{array}{l}\text { Escritura - Preparación del } \\
\text { borrador original }\end{array}$ & \begin{tabular}{l} 
José Ignacio Aguilar Carrasco \\
\hline $\begin{array}{l}\text { Escritura - Revisión } \\
\text { edición }\end{array}$
\end{tabular}$\quad \begin{array}{l}\text { José Ignacio Aguilar Carrasco «igual», Roberto Arvizu Acosta } \\
\text { «igual», Ângel Evaristo Flores Ramírez «igual» }\end{array}$ \\
\hline $\begin{array}{l}\text { Visualización } \\
\text { Supervisión }\end{array}$ & $\begin{array}{l}\text { José Ignacio Aguilar Carrasco «igual», Roberto Arvizu Acosta } \\
\text { «apoyo» }\end{array}$ \\
\hline $\begin{array}{l}\text { Administración } \\
\text { Proyectos }\end{array}$ & José Ignacio Aguilar Carrasco \\
\hline Adquisición de fondos & José Ignacio Aguilar Carrasco \\
\hline
\end{tabular}

